University of Wollongong

Research Online

Faculty of Engineering - Papers (Archive)

Faculty of Engineering and Information

Sciences

$1-1-2007$

\title{
A flexible approach to on-site power filtering and supply backup using high capacity high temperature SMES for maximum equipment utility
}

\author{
Dominic Cuiuri \\ University of Wollongong, dominic@uow.edu.au \\ Jeffrey W. Moscrop \\ University of Wollongong, jeffm@uow.edu.au \\ Christopher David Cook \\ University of Wollongong, chris_cook@uow.edu.au
}

Follow this and additional works at: https://ro.uow.edu.au/engpapers

Part of the Engineering Commons

https://ro.uow.edu.au/engpapers/1082

\section{Recommended Citation}

Cuiuri, Dominic; Moscrop, Jeffrey W.; and Cook, Christopher David: A flexible approach to on-site power filtering and supply backup using high capacity high temperature SMES for maximum equipment utility 2007, 601-606.

https://ro.uow.edu.au/engpapers/1082

Research Online is the open access institutional repository for the University of Wollongong. For further information contact the UOW Library: research-pubs@uow.edu.au 


\title{
A Flexible Approach to On-Site Power Filtering and Supply Backup using High Capacity High Temperature SMES for Maximum Equipment Utility
}

\author{
Dominic Cuiuri, Jeff Moscrop and Chris Cook
}

\begin{abstract}
With power systems subject to an ever-increasing variety of load types, power quality remains of concern to both utilities and consumers alike. Some of the most concerning issues to users of sensitive equipment include voltage sags, supply interruption and current harmonics. This paper describes a comprehensive power quality device that can be designed to compensate for harmonic load currents and mains voltage variations in a system, as well as providing mains failure backup for smaller critical system loads. The design process described integrates high capacity high temperature Superconducting Magnetic Energy Storage (SMES) technology with Active Power Filter (APF) switching technology ySMES devices to suit critical load power requirements in a system and the complete design philosophy that maximises the usefulness of the whole power conditioning system are both discussed in the paper.
\end{abstract}

\section{INTRODUCTION}

The demand for improved power quality has resulted in the development of a range of power conditioning devices, including constant voltage transformers, static voltage compensators, tap changing regulators, Uninterruptible Power Supplies (UPS) and Active Power Filters (APF). One example of a comprehensive power conditioning device, that can compensate for current harmonics, voltage errors and complete supply interruptions, has been studied in [1]. This particular device uses the combination of UPS and APF technology to achieve comprehensive power conditioning.

A new power conditioning device is currently being developed at the University of Wollongong. Like the design presented in [1], this new device uses both shunt and series APFs; however, instead of a UPS the device utilises the inherent advantages of high transition temperature Superconducting Magnetic Energy Storage (SMES) technology. The superconducting coil of the SMES is wound using High Temperature Superconducting (HTS) tape and does not require maintenance. The SMES coil also has almost zero losses and has a much higher energy density than batteries above a certain size, making it much more suited for large applications. Furthermore, as the energy is stored electrically, there is no electrochemical conversion necessary and consequently the response time is faster than battery based systems.

The complete design of the SMES/APF power conditioning device is discussed in this paper, including sizing of the SMES to suit the critical system load requirements and the concept of coupling the SMES with APF technology to maximise the usefulness of the power conditioning system.

\section{SMES DESIGN}

The three important electrical specifications of a superconducting coil are the energy capacity, the current available at maximum energy storage and the inductance. The energy capacity $\left(\mathrm{E}_{\max }\right)$ of the SMES system considered in this paper is described by Equation (1), where $\mathrm{P}_{\text {critical }}$ is the total power requirement of the critical system loads and $t_{\text {holdup }}$ is the required hold-up time during a mains failure.

$$
\mathrm{E}(J)=\mathrm{P}_{\text {critical }}(W) \times t_{\text {holdup }}(s)
$$

In order to determine the current at maximum energy storage, the ratio between the energy that remains in the coil at the load balance point and the total energy capacity of the coil must be specified $\left(\mathrm{K}_{e}=\mathrm{E}_{\text {balance }}: \mathrm{E}_{\text {max }}\right)$. The load balance point is the point where the coil can no longer support the full power requirements of the critical load. Defining $i_{\text {balance }}$ as the current drawn by the critical load and $i_{\text {standby }}$ as the current available at maximum energy storage:

$$
\begin{gathered}
\mathrm{E}_{\text {balance }}=\frac{1}{2} \mathrm{~L} i_{\text {balance }}^{2} \\
\mathrm{E}_{\max }=\frac{1}{2} \mathrm{~L}_{\text {standby }}^{2} \\
\text { Hence: } i_{\text {standby }}=\frac{i_{\text {balance }}}{\sqrt{\mathrm{K}_{e}}}
\end{gathered}
$$

The coil inductance can be determined by substituting Equations (1) and (4) into Equation (3). The final expression for coil inductance is given in Equation (5).

$$
\mathrm{L}=\frac{2 \mathrm{~K}_{e} \mathrm{P}_{\text {critical }} \text { holdup }_{\text {p }}}{i_{\text {balance }}^{2}}
$$

A small value of $\mathrm{K}_{e}$ is chosen when the superconducting coil is designed for high energy utilisation; hence, $i_{\text {standby }}$ can be considerably higher than $i_{\text {balance }}$ (as per Equation (4)). Through the APF design, the larger $i_{\text {standby }}$ current is available for harmonic correction. Since the ratio of fundamental current to harmonic current is usually high, the resulting SMES/APF power conditioning device has the potential capability of 
providing mains backup for critical loads and compensating for the harmonics of much higher non-critical loads.

As an example, consider a SMES superconducting coil that is required to provide mains backup of a $10 \mathrm{~kW} 3$-phase critical load for a period of 2 seconds. From Equation (1) the energy capacity of the SMES system would need to be $20 \mathrm{~kJ}$. The average DC bus linkage voltage is:

$$
\frac{3 \times 415 \sqrt{2}}{\pi} \approx 560 \mathrm{VDC}
$$

Hence, the minimum coil current required to balance the load is:

$$
i_{\text {balance }}=\frac{10 \mathrm{~kW}}{560 \mathrm{v}} \approx 17.9 \mathrm{~A}
$$

If the coil is designed with $\mathrm{E}_{r}=0.1$ (90\% energy utilisation during mains failure), the current available at maximum energy storage is 56.6A (Equation (4)). Also, from Equation (5), the coil inductance would be approximately $12.5 \mathrm{H}$. Hence, the electrical specifications of the superconducting coil are $20 \mathrm{~kJ}$, $56.6 \mathrm{~A}$ and $12.5 \mathrm{H}$.

Through the APF function, this example coil would have the capability of supplying 40A RMS of harmonic correction current. If it is assumed that the ratio of RMS fundamental current to harmonic current is 5:1, the coil could correct for harmonics in a 200A RMS system. Hence, the complete power conditioning device would have the capability to provide mains backup of the $10 \mathrm{~kW}$ critical loads for 2 seconds and also to compensate for harmonic currents of a 144kVA non-critical system $(\sqrt{3} \times 415 \times 200=144 \mathrm{kVA})$.

The design and construction of a prototype $2.5 \mathrm{~kJ}$ HTS SMES device has previously been undertaken at the University of Wollongong [2], [3]. Although the superconducting coil of this prototype was designed to have a $2.5 \mathrm{~kJ}$ energy capacity, many of the other system components were sized to suit higher capacity SMES systems and have been retained for the SMES/APF power conditioning device currently under development.

One of the components retained in the current project is the cryogenic cooling system, which uses the conduction cooling method and consists of both a cryocooler and a cryostat. A single stage cold head, supplied by a gaseous helium compressor, is used in the cryocooler system. The cryostat houses the superconducting coil in a vacuum chamber and has radiation baffles, super insulation wrapping and a liquid nitrogen buffer to reduce radiative heat loss. The cryostat is shown in Fig. 1.

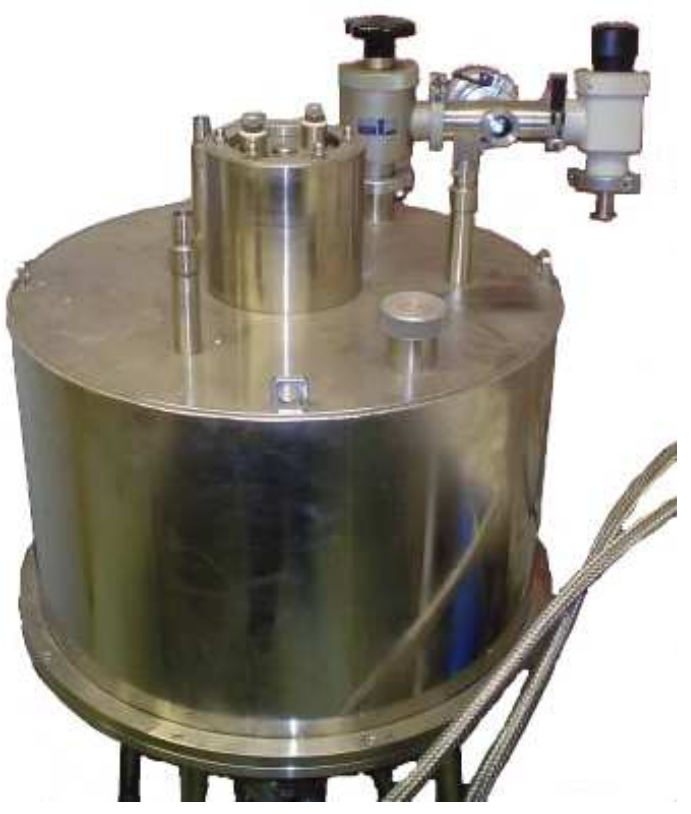

Fig. 1. SMES System Cryostat

The cold head of the cryocooler is mounted on the underside of the cryostat, as shown in Fig. 2.

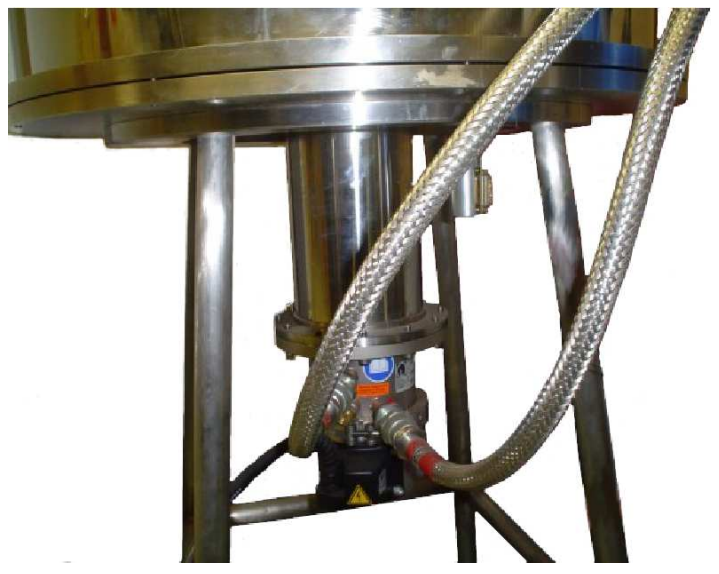

Fig. 2. The Cryocooler Cold Head

The superconducting coil of the $2.5 \mathrm{~kJ}$ prototype device was designed in a solenoid fashion using Finite Element Modelling (FEM). The coil former was made from copper and was positioned inside the cryostat on a cold plate that provided direct thermal contact with the cryocooler cold head. The design process that is currently being undertaken on a new high capacity superconducting coil is focused on improving both the electrical specifications and the thermal characteristics of the coil and former.

\section{Power Conditioning System Design}

The system currently under development integrates series and parallel APFs, SMES storage and a means of load segregation to provide a comprehensive power conditioning system. A 
block diagram of this power conditioning system is given in Fig. 3.

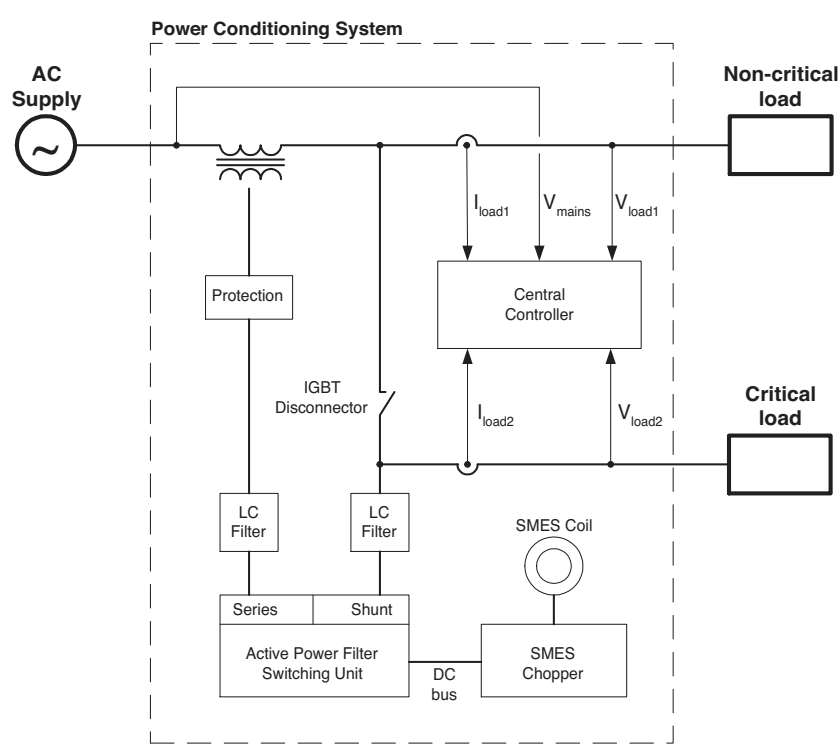

Fig. 3. Power Conditioning System

Depending on the application, a power conditioning system can be designed to meet some or all of the following disturbances:

\section{- Line Voltage Disturbances}

V1. Long term frequency variation

V2. Medium to long term RMS surge ( $>1$ cycle)

V3. Medium to long term RMS sag ( $>1$ cycle)

V4. Transient over-voltage $(<1$ cycle $)$

V5. Transient under-voltage $(<1$ cycle $)$

V6. Phase Voltage imbalance

V7. Medium to long term harmonics

V8. Short term complete interruption

V9. Long term complete interruption

- Line Current Disturbances

C1. Non-unity power factor

C2. Low frequency harmonics (from load)

Parallel active power filters act as a controllable current source in parallel with the load. These filters are able to compensate for undesirable line current disturbances that are introduced onto the mains by a "non-ideal" load. These include items $\mathrm{C} 1$ and C2. Parallel filters are unable to significantly correct the mains terminal voltage at the point of common coupling, except through the effect of minor voltage changes due to the effective line impedance up to the point of common coupling. Furthermore, the parallel APF has no major energy storage components, so the average real power drawn from the supply must be zero (except for losses). This imposes constraints on the compensation current waveforms that can be drawn from the supply by the parallel APF.

Series active power filters act as a controllable voltage source in series with the mains supply. Consequently, these filters are able to significantly modify the supply terminal voltage.
Compensation for disturbances V2 to V7 is possible, within the limitations of the filter response time. There is a tradeoff between the amount of compensation available and the component ratings, hence filter cost. Unlike the parallel APF, the series APF allows for more (or less) real power to be drawn from the supply by the load. The series APF can be considered to act as a rapidly adjustable autotransformer, independently adjustable for each phase of a multiphase system.

Conditions V1 and V9 cannot be corrected by APF technology and require a UPS topology for correction. However, UPS systems are often prohibitively expensive and unnecessary for many loads. For the other conditions, combined series/parallel active power filters can achieve the equivalent filtering capability of UPS at lower cost. The reactive power compensating capacity of the filter is usually less than $20 \%$ of the real power requirement of the load. This has provided a major impetus for APF development.

The additional concept of segregating the total load into critical and non-critical loads [1] allows the system designer to bridge the gap between APF and UPS functional capabilities. Introducing a modest energy storage component into the system allows for the critical load to be protected against short-term supply interruption at low additional cost to the system. Where the power requirement of the critical load is a small fraction of the non-critical conditioned load, a modest energy storage system can provide significant backup time.

Implementation of this power conditioning system requires a device to rapidly disconnect the non-critical load from the critical load, so that the parallel (or shunt) APF is not overloaded and a minimum of energy is expended in the noncritical load. In previous developments [1], the disconnection has been done with thyristors. These are inexpensive, but the disconnection time may reach 10 milliseconds ( $\frac{1}{2}$ mains cycle). For critical industrial loads such as those that use latching contactors or relays in safety circuits, this is unacceptably long and would cause loss of process control. The use of IGBTs for the disconnecting element is expected to overcome the disadvantage of thyristors, with only a small cost increase.

The additional challenges that are presented by this particular power conditioning system over other topologies are:

1) Reliable and rapid detection of supply loss, to make full use of the high speed disconnection capability.

2) Seamless changeover of parallel APF PWM output from parallel current compensation to sinusoidal mains voltage supply, and vice versa.

Fig. 4 shows a more detailed main circuit of the power conditioning system that is currently under development. The design is based on a single phase module which can be duplicated to service a three phase application. The central controller utilises a fixed point DSP (Motorola 56F807). The six IGBTs required by the series APF utilise a 6-pack intelligent power module. The parallel APF is implemented with a dual-pack IGBT module. The elements required for regulation of the SMES coil current are two "chopper" modules (one diode and one IGBT in each module). The disconnecting element is constructed 
with two back-to-back IGBTs (single-pack isolated modules) Where and associated snubber components (not shown).

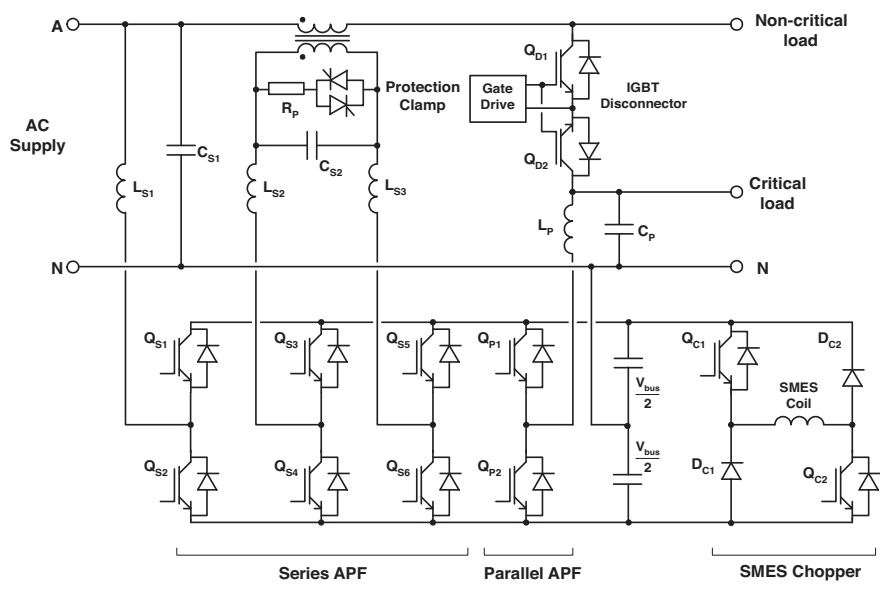

Fig. 4. Power Conditioning System Main Circuit

\section{A. Parallel APF Components}

The ratings of the key components are subject to the load characteristics, It is instructive to examine one particular type of load to get an indication of the component rating relative to the load rating. A particularly poor load is a large three phase 6 pulse diode rectifier with highly inductive DC side filter and resistive load (Fig. 5). This is a severe application, as it draws almost a square wave of current from its supply. Thyristor-based drives for large DC motors exhibit this type of input current waveform at full load.

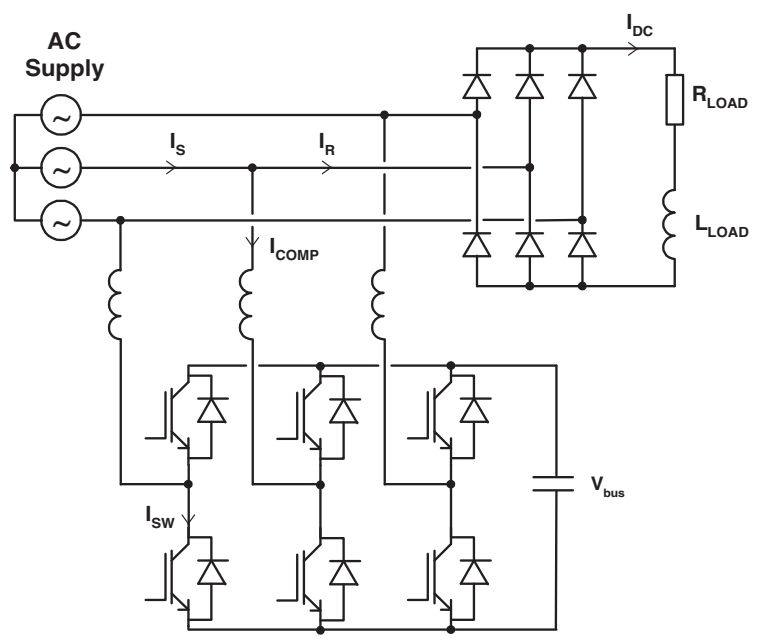

Fig. 5. Parallel APF Applied to 6 Pulse Diode Rectifier

Analysis of a parallel APF used to compensate such a load [4] shows that the mean current through any one switching element (IGBT combined with flywheel diode) is related to the RMS rectifier current by:

$$
I_{s w, a v g}=\frac{3 \sqrt{2}}{\pi^{2}} I_{R, R M S}\left[1-\sqrt{3}+2 \cos \phi+\frac{\pi}{\sqrt{3}}\left(\phi-\frac{\pi}{3}\right)\right]
$$

$$
I_{R, R M S}=\frac{\sqrt{2}}{\sqrt{3}} I_{d c}
$$

And

$$
\phi=\sin ^{-1}\left(\frac{\pi}{2 \sqrt{3}}\right)
$$

After compensation, the supply current $I_{S}$ will be the fundamental component of the rectifier line current. The RMS value is:

$$
I_{S, R M S}=\frac{\sqrt{6}}{\pi} I_{d c}
$$

Equation (6) can be numerically evaluated:

$$
I_{s w, a v g}=0.117 I_{R, R M S}
$$

The average current rating of the parallel APF switching element is approximately $12 \%$ of the RMS current rating of a 6 pulse rectifier-type load. For a $415 \mathrm{~V}$ L-L supply, a 140kW DC load will require $250 \mathrm{~A}$ of current at $560 \mathrm{Vdc}$, so the AC RMS load current will be 204A. The parallel APF IGBT switch element will handle an average current of only 24A while providing full harmonic compensation for this $147 \mathrm{kVA}$ load. The RMS value of the sinusoidal current drawn from the mains will be 195A.

It should be noted that when selecting the devices to be used, the apportionment of conduction losses between IGBT and flywheel diode need to be assessed by calculating the average current through each device. The "sharing" of average current between IGBT and diode will be influenced by the current compensation waveform and its relationship to the mains voltage waveform: The instantaneous duty cycle of the PWM waveform that is required to produce the compensating current will alter the per-PWM-cycle distribution of current between IGBT and diode. Furthermore, the switching losses incurred by the IGBT and flywheel diode also need to be included. This is best done by using an appropriate circuit simulation software package whilst modelling the worst-case load situation.

\section{B. Disconnecting Element Components}

The compensating current applied to the non-critical load by the parallel APF must be conducted by the disconnecting element. It must also conduct the load current drawn by the critical load from the supply during normal operation. Considering just the parallel APF compensation current component, the average current in each IGBT and diode of the disconnecting element is the same as the average current in the switching element of the parallel APF (Equations (6) and (10)). Unlike the parallel APF, there are no switching losses during normal operation, so thermal calculations are greatly simplified.

The contribution of the critical load to the disconnecting element average current should be taken into account before the integration that leads to Equation (6). The result will of course be different for various types of load current waveforms. 
For the purposes of selecting components, a simplifying and conservative approximation may be made. As stated in Section 2 , assuming that the critical 3 phase load is balanced and requires $10 \mathrm{~kW}$, the RMS line current is approximately $13.9 \mathrm{~A}$ for unity power factor. The half-rectified sinewave current conducted by each IGBT and diode is:

$$
I_{C R I T, \text { half-rect }}=\frac{\sqrt{2} I_{R M S}}{\pi}=0.45 \times 13.9=6.3 \mathrm{~A}
$$

Adding this to the parallel APF average current results in a total average current of 30A for both IGBT and diode. The contribution of a small critical load only marginally increases the current rating of the disconnecting element components.

\section{Series APF Components}

The series APF is used to perform supply voltage waveform corrections. As the parallel APF ensures that the supply current is sinusoidal, the current delivered to the series APF transformer will also be sinusoidal, and related to the load current by the turns ratio $(n)$. To perform the voltage correction, the PWM voltage source inverter (VSI) output of the series-APF bridge needs to be filtered and applied to the "secondary" of the transformer. The transformer turns ratio is dictated by:

1) The maximum desired voltage correction

2) The maximum available voltage output of the PWM VSI.

3) Protection requirements of the PWM VSI components during short-circuit fault conditions.

A reasonable specification for RMS mains voltage correction is $\pm 10 \%$. The maximum fundamental sinusoidal output voltage of the PWM VSI is limited by the available DC bus voltage and the maximum modulation depth of the PWM modulation scheme. The DC bus voltage is shared with the parallel APF, and is usually $15 \%$ above the peak rectified mains voltage so that output current control is still possible at the peak mains voltage. This voltage boost function can be performed by the parallel APF, which has the necessary topology. Alternatively, a dedicated input stage for the series APF can be used $\left(Q_{S 1}\right.$ and $\mathrm{Q}_{S 2}$ in Fig. 4) which draws sinusoidal in-phase current directly from the unmodified mains supply.

The peak output voltage of the series APF VSI is:

$$
V_{p k}=M_{\max } \frac{V_{b u s}}{2}
$$

where $M_{\max }$ is the maximum inverter modulation depth. The corresponding fundamental RMS output voltage that is applied to the transformer "secondary" is:

$$
V_{R M S}=\frac{M_{\max } V_{\text {bus }}}{2 \sqrt{2}}
$$

The transformer turns ratio is then evaluated as:

$$
n=\frac{V_{s e c}}{V_{p r i}}=\frac{V_{R M S}}{x V}
$$

where $x$ is the maximum percentage correction that needs to be applied to the mains voltage. For example, if the maximum modulation depth is 0.9 , the DC bus voltage is $750 \mathrm{~V}$, the mains voltage is $240 \mathrm{~V}$ and a $10 \%$ correction is required, then:

$$
n=\frac{\frac{0.9 \times 750}{2 \sqrt{2}}}{0.1 \times 240}=9.96
$$

It is assumed that the parallel APF compensates for the load harmonic currents, so the current flowing through the series APF transformer "primary" is sinusoidal and in phase with the mains voltage. The analysis of currents within the series APF becomes similar to the case for a conventional AC drive inverter operating at a unity power factor output.

The IGBT duty cycle varies through the positive fundamental half-cycle as:

$$
\delta_{I G B T}(\theta)=\frac{1+M \sin (\theta)}{2}
$$

where $M$ is the modulation depth (between 0 and 1). Equation (16) is valid for values of $\theta$ from zero to $\pi$. The flywheel diode duty cycle is the complement of the IGBT duty cycle:

$$
\delta_{F W D}(\theta)=1-\delta_{I G B T}(\theta)=\frac{1-M \sin (\theta)}{2}
$$

At unity power factor, the inverter output current is in phase with the fundamental output voltage of the inverter. It can be expressed as:

$$
i(\theta)=\sqrt{2} I \sin (\theta)
$$

where $I$ is the secondary-referred RMS load current. The average current through the IGBT is evaluated from:

$$
\begin{gathered}
I_{I G B T, \text { avg }}=\frac{1}{2 \pi} \int_{0}^{\pi}\left[\sqrt{2} I \sin (\theta) \times \frac{1}{2}(1+M \sin (\theta))\right] d \theta \\
I_{I G B T, \text { avg }}=\frac{\sqrt{2} I}{4 \pi}\left(2+\frac{\pi M}{2}\right)
\end{gathered}
$$

The IGBT switching losses can be estimated from the average current and the switching frequency:

$$
P_{S W, I G B T}=\left(E_{O N}+E_{O F F}\right) f_{S W}
$$

where $E_{O N}$ and $E_{O F F}$ are obtained from the manufacturer's data sheet, evaluated at the operating bus voltage and average current. This approximation is valid because the switching losses (turn-on and turn-off) are proportional to the current, so the average current will reflect the average switching loss.

The average flywheel diode current can be evaluated in the same manner as the average IGBT current:

$$
\begin{gathered}
I_{F W D, \text { avg }}=\frac{1}{2 \pi} \int_{0}^{\pi}\left[\sqrt{2} I \sin (\theta) \times \frac{1}{2}(1-M \sin (\theta))\right] d \theta \\
I_{F W D, \text { avg }}=\frac{\sqrt{2} I}{4 \pi}\left(2-\frac{\pi M}{2}\right)
\end{gathered}
$$

When the average IGBT and flywheel diode currents are summed, the result is the total switching element current:

$$
I_{S W, a v g}=\frac{\sqrt{2} I}{\pi}
$$

This corresponds to the average value of a half-rectified sinewave of RMS value I, as would be expected. 
Continuing the example of the 6 pulse rectifier load from Section III-A; with a 195A RMS sinusoidal primary current and a 10:1 transformer ratio, $\mathrm{I}$ is $19.5 \mathrm{~A}$. For a maximum modulation depth of 0.9 , the average IGBT current is $7.49 \mathrm{~A}$ $\left(\mathrm{Q}_{S 3}\right.$ to $\mathrm{Q}_{S 6}$ in Fig. 4). Note that the peak IGBT current is 27.6A under normal operating conditions, so the device needs to be sized accordingly. The average flywheel diode current is $1.29 \mathrm{~A}$. Assuming a saturation voltage of $3 \mathrm{~V}$, the IGBT conduction losses are approximately $23 \mathrm{~W}$. From the data sheet of a $35 \mathrm{~A} 1200 \mathrm{~V}$ device, values for $E_{O N}$ and $E_{O F F}$ are interpolated at $0.94 \mathrm{~mJ}$ and $1.26 \mathrm{~mJ}$ respectively, for $7.5 \mathrm{~A}$ and $750 \mathrm{~V}$. The switching losses at $20 \mathrm{kHz}$ are approximately $44 \mathrm{~W}$. In relation to the power rating of the load, these device ratings are quite modest.

As mentioned earlier, the power that is required to perform the supply voltage correction is supplied to the DC bus by components $\mathrm{Q}_{S 1}$ and $\mathrm{Q}_{S 2}$. The current drawn through inductor $\mathrm{L}_{S 1}$ is either in phase with the supply voltage when a voltage increase is required, or 180 degrees out of phase in the case where a voltage reduction is necessary. Ignoring losses, the RMS input current to the APF is found by applying the conservation of power rule:

$$
I_{S A P F, \text { input }}=x I_{L O A D, R M S}=0.1 \times 195=19.5 \mathrm{~A}
$$

The PWM modulation scheme used to regulate this input current produces similar waveforms to that required for regulation of the output voltage of the series APF, so Equations (20) and (23) can be used to find the average currents for $\mathrm{Q}_{S 1}$ and $\mathrm{Q}_{S 2}$. In the case of a $750 \mathrm{~V}$ DC bus and a peak supply voltage of $306 \mathrm{~V}$ ( $10 \%$ down from the nominal peak value), the modulation depth $M$ is approximately 0.82 . The average IGBT and diode currents are 7.2A and 1.6A respectively. These are very similar to the currents calculated for $\mathrm{Q}_{S 3}$ to $\mathrm{Q}_{S 6}$.

Practical methods for protection of the VSI are discussed in [5]. The basic requirements are to prevent the transformer "secondary" voltage from exceeding the DC bus supply rails during a supply overcurrent fault, so that the VSI is not exposed to output overvoltages that produce uncontrolled currents in the switching components. One method is to switch in a low resistance value $\left(\mathrm{R}_{p}\right.$ in Fig. 4) across the transformer "secondary" when a mains overcurrent condition is detected. The VSI is disabled during the fault condition. The transformer is also designed such that magnetic saturation occurs rapidly under fault conditions, so the duration of secondary winding fault current is limited to short time intervals around the current zero-crossing points [5].

\section{SMES Chopper Components}

For the vast majority of its operation, the SMES chopper circuit shown in Fig. 4 circulates standby current through the SMES coil using components $\mathrm{Q}_{c 1}$ and $\mathrm{D}_{c 2}$. These components must be continuously rated for the SMES standby current. Using the example in Section 2 (56.6A), and assuming IGBT and diode conduction voltages of $2.5 \mathrm{~V}$ and $1.1 \mathrm{~V}$ respectively, the conduction losses will be $142 \mathrm{~W}$ and $62 \mathrm{~W}$ respectively.
These devices have a higher duty in comparison with their counterparts in the APFs.

Devices $\mathrm{Q}_{c 2}$ and $\mathrm{D}_{c 1}$ conduct during SMES charging and discharging, respectively. In the case of charging, the duty cycle is extremely low, and can be estimated by:

$$
\delta_{Q c 2}=\frac{V_{\text {cond }}}{V_{\text {cond }}+V_{\text {bus }}}
$$

where $V_{\text {cond }}$ is the sum of the IGBT saturation voltage, flywheel diode forward drop, and any other incidental voltage drops in the circuit. The switching frequency of the chopper while regulating the SMES standby current is approximately:

$$
f_{S W}=\frac{V_{\text {cond }} V_{\text {bus }}}{L \Delta I\left(V_{\text {cond }}+V_{\text {bus }}\right)}
$$

where $\Delta I$ is the hysteresis band used to regulate the SMES standby current and $L$ is the SMES coil inductance. Continuing the examples from Sections 2 and 3.3, if the SMES coil inductance is $12.5 \mathrm{H}, V_{\text {cond }}$ is $3.6 \mathrm{~V}$, the DC bus voltage is $750 \mathrm{~V}$, and $\Delta I$ is $2 \mathrm{~A}$, then the duty cycle of $\mathrm{Q}_{c 1}$ is $99.47 \%$, the duty cycle of $\mathrm{Q}_{c 2}$ is $0.53 \%$, and the switching frequency is approximately $0.16 \mathrm{~Hz}$. Under these conditions, the device losses are dominated by conduction losses.

\section{CONCLUSIONS}

The design aspects of a comprehensive power quality device are described in this paper. A high capacity SMES coil and the related cooling system have been described, with reference to a previously built $2.5 \mathrm{~kJ}$ prototype. A power conditioning system, which integrates SMES and APF technologies to compensate for harmonic load currents and mains voltage variations for all system loads, as well as providing mains failure backup for smaller critical loads in a system, has also been discussed in detail. As can be seen from the calculations, the average current rating of semiconductor devices is small in comparison to the rating of the compensated non-linear load. The segregation of critical and non-critical loads allows for extended backup times from a modest energy storage system. These two features provide an economical and comprehensive power conditioning system for many applications.

The work described in this paper is jointly funded by the Australian Research Council and Australian Superconductors Pty Ltd.

\section{REFERENCES}

[1] F. Barrero, S. Martinez, F. Yeves, F. Mur, and P. Martinez. Universal and reconfigurable to ups active power filter for line conditioning. IEEE Transactions on Power Delivery, 18:283-290, 2003.

[2] C.J. Hawley. Design and Manufacture of a High Temperature Superconducting Magnetic Energy Storage Device. $\mathrm{PhD}$ thesis, The University of Wollongong, Wollongong, Australia, 2005.

[3] C.J. Hawley and S.A. Gower. Design and preliminary results of a prototype hts smes device. IEEE Transactions on Applied Superconductivity, 15:1899-1902, 2005.

[4] J.H. Marks and T.C. Green. Ratings analysis of active power filters. IEE Proceedings on Electric Power Applications, 150:607-614, 2003.

[5] L. Moran, I. Pastorini, J. Dixon, and R. Wallace. A fault protection scheme for series active power filters. IEEE Transactions on Power Electronics, 14:928-938, 1999. 